\title{
L'ILLUMINISMO DELLE RIFORME CIVILI: DIVISIONE DEL LAVORO, COMMERCIO, PRODUZIONE DELLA RICCHEZZA
}

\author{
ROBERTO SCAZZIERI (*)
}

\begin{abstract}
RIASSUNTO. - La formazione dell'economia politica è strettamente collegata alle tradizioni intellettuali dell'illuminismo. Tuttavia l'illuminismo degli economisti è un fenomeno complesso di cui è importante non dimenticare la pluralità di articolazioni. Non diversamente da quello che accade per l'lluminismo in generale, anche l'illuminismo degli economisti si esprime attraverso analisi e proposte spesso molto lontane fra loro, anche se non è difficile individuarne una matrice comune. Ad esempio, l'illuminismo di coloro che, come i Fisiocrati, assumono il punto di vista dei "consiglieri esterni" rispetto all'autorità sovrana è profondamente diverso dalla prospettiva degli economisti che adottano l'ottica dei "commercianti colti" e sottolineano l'autonomia relativa di divisione del lavoro e mercati rispetto agli stati; così come è diverso dalla prospettiva di quegli economisti che si collocano per così dire in una posizione intermedia fra mercati e strutture di governo e ne esplorano le interdipendenze sul piano analitico e su quello dell'azione politico-amministrativa.

La riflessione economica degli illuministi lombardi si caratterizza per la capacità di visualizzare, all'interno di uno stesso sistema di analisi e di proposte, sia il punto di vista "orizzontale" della divisione del lavoro e degli scambi sia la prospettiva "verticale" degli apparati amministrativi. Si delinea in questo modo uno schema di economia politica e di governo dell'economia che è lontano dall'illuminismo radicale di Condorcet, Morellet, Paine, così come è lontano dal punto di vista del laisser faire "dirigista" di Quesnay e della sua scuola, e dal riformismo più aperto a interventi mirati di Genovesi, Galiani e Tanucci. Il duplice inserimento di molti dei suoi esponenti (e in primo luogo di Cesare Beccaria e Pietro Verri) all'interno della classe dirigente milanese ma anche nelle strutture di governo dell'autorità centrale rende il loro contributo di particolare interesse. L'obiettivo principale di questa relazione è discutere il contributo degli illuministi lombardi alla defini-
\end{abstract}

(*) Università di Bologna e Istituto Lombardo Accademia di Scienze e Lettere. Relazione presentata al convegno "L'Illuminismo delle riforme civili: il contributo degli economisti lombardi”, Istituto Lombardo Accademia di Scienze e Lettere, Milano, 1314 dicembre 2011. 
zione di un corpus di economia politica caratterizzato dalla sovrapposizione fra punto di vista della società commerciale e punto di vista degli apparati di governo (sovrapposizione che in questo convegno abbiamo inteso caratterizzare con l'espressione di illuminismo civile). I risultati di questo intreccio sono spesso di grande interesse per quanto riguarda la comprensione dei nessi tra divisione del lavoro e mercati, ordinamemento giuridico e governo dell'economia. Come si è prima osservato, divisione del lavoro e mercati costituiscono ambiti privilegiati di attenzione per economisti che assegnano alle capacità di iniziativa di individui e gruppi un'efficacia autonoma rispetto alle decisioni politiche e alle loro conseguenze sul piano amministrativo. Questo spiega la centralità rispettiva di divisione del lavoro e commerci negli scritti economici di Beccaria e Verri. In particolare, il punto di vista dell'illuminismo civile suggerisce immediatamente la necessità di inserire la divisione del lavoro in un tessuto complesso di organizzazione "gerarchica" delle strutture produttive (Beccaria); lo stesso punto di vista indica l'esigenza di studiare commerci e mercati mettendo in risalto architetture istituzionali e configurazioni amministrative piuttosto che situazioni e criteri di scelta derivati per via inferenziale da principi di razionalità astratta (Verri).

In questa prospettiva, assume un ruolo centrale l'analisi delle azioni di governo intese come interventi "interni" alle stesse strutture della divisione del lavoro e dei mercati, e proprio per questa ragione efficaci a sollecitare e orientare capacità già esistenti all'interno del tessuto sociale. Diritto, amministrazione e politica monetaria configurano un quadro di analisi e di proposte che colloca al centro i processi di formazione della ricchezza e li considera parte integrante della struttura sociale nelle sue articolazioni istituzionali e amministrative. Si delinea in questo modo un insieme di implicazioni reciproche fra economia, politica e società che costituisce l'aspetto più caratteristico dell'illuminismo delle riforme civili.

\section{AbSTRACT. - The Enlightenment of Civil Reforms: Commerce, Division of Labour, and the Production of Wealth.}

The formation of political economy is closely associated with the intellectual traditions of the enlightenment. However, the economists' enlightenment is a complex process characterized by a plurality of roots and expressions. It encompasses analyses and policy proposals remarkably distant from each other and yet pointing to a common matrix of concepts and beliefs. To take one example, the enlightenment of the Physiocrats, who take the standpoint of 'external advisors' to the Sovereign, is different from the enlightenment of those 'cultivated traders' who emphasize the relative autonomy of division of labour and markets relative to the state; as it is different from the standpoint of economists who focus on the middle ground between markets and governmental structures and explore their interdependence both at the level of analysis and at that of administrative and political action. The contributions of the Lombard Enlightenment are characterized by the ability to visualize both the 'horizontal' standpoint of division of labour and exchange, and the 'vertical' perspective of administrative structures. In this way, the Lombard economic enlightenment brings into focus a scheme of economic analysis and economic governance that is distinct from the radical Enlightenment of Condorcet, Morellet and Paine, as it is distinct from the topdown' laisser faire of Quesnay and Physiocracy in France, and from Genovesi's, Galiani's and Tanucci's more active and context-dependent approach to economic policy in Naples. Many economists of the Lombard Enlightenment, such as Cesare 
Beccaria and Pietro Verri, were part of the Milanese social elite but were also active in the administrative structures of government. This makes their intellectual and policy activity of special interest. The principal objective of this paper is to discuss the contribution of the Lombard Enlightenment to the definition of a body of economic knowledge in which it is possible to see a clear overlap. between the point of view of commercial society and that of governmental structures (an overlap. that is characteristic of the intellectual make up. of civil enlightenment). This overlap. is of distinctive importance for investigating the relationship. between division of labour and markets, legal arrangements, and governance of the economy. Division of labour and markets are central to economists considering the initiatives of individuals and groups independently of political decisions and of their administrative consequences. This explains the central position of trade and division of labour in the writings of Beccaria and Verri. However, the perspective of the civil enlightenment suggests including division of labour within a complex organizational hierarchy of productive activities (Beccaria). The same point of view also calls attention to the need of investigating commerce and markets by focusing on institutional and administrative conditions rather than on choice criteria derived from abstract rationality principles (Verri). The Lombard Enlightenment economists emphasize the central position of governmental actions as external interventions with respect to division of labour and markets, and for this reason capable of triggering the activation of possibilities existing within a given social context. Law, administration and monetary policy bring about an analytical and policy framework focusing on the process of wealth formation and considering this process as embedded in the institutional and administrative arrangements of society. In this way, the economists of the Lombard Enlightenment emphasize the mutual relationship. between administrative governance, economic improvement, and social structures, and take this relationship. to be at the core of the enlightenment of civil reforms.

\section{PREMESSA}

L'illuminismo economico di Milano e della Lombardia è uno dei momenti più significativi del Settecento riformatore in Europa. Con l'illuminismo napoletano esso costituisce il contributo più alto della cultura italiana allo sviluppo di una moderna tradizione europea di riforme civili e governo dell'economia.

Gli economisti lombardi condividono l'interesse dell'illuminismo francese per un'attività razionale di governo e quello dell'illuminismo scozzese per le configurazioni e le dinamiche della società civile. Tuttavia, esso si distingue da entrambi per l'attenzione rivolta alle connessioni fra legislazione, riforme e cultura civile. Si distingue da essi anche per essere parte del complesso intreccio di reciproche influenze che caratterizza il microcosmo degli stati e città italiane nel Settecento. Gli scritti economi- 
ci degli illuministi lombardi assumono la socievolezza come punto di partenza ma considerano la stessa come intrinsecamente connessa a tradizioni civili (reali o immaginate), e la trovano espressa in modo prioritario attraverso la divisione del lavoro, la produzione della ricchezza e le interdipendenze fra mercati. Alcuni dei più importanti contributi dell'illuminismo lombardo studiano gli assetti economici e le disposizioni amministrative di una società che si governa in modo autonomo. In questa prospettiva, economia, giustizia e legislazione acquistano una posizione centrale, e si evitano sia l'enfasi degli illuministi scozzesi sulla conformità spontanea nella commercial society sia quella degli illuministi francesi sul disegno razionale delle politiche economiche e amministrative. Il coordinamento sociale è preso in esame a partire dagli ordinamenti civili esistenti e attraverso la griglia interpretativa fornita da alcuni prototipi di filosofia politica e morale. Tutto questo conduce ad una caratteristica combinazione di ambizioni teoriche e preoccupazioni pratiche e spiega l'interesse degli illuministi lombardi per questioni come il commercio e i dazi, la politica monetaria, la tassazione fondiaria, e le regole sull'organizzazione della produzione.

Come ebbe a notare Franco Venturi, la tensione fra utopia e riforma è centrale nell'illuminismo italiano e spiega la maggior parte dei suoi principali contributi (Venturi, 2001, pp. 29-59). In questa prospettiva, l'illuminismo lombardo si distingue per il suo carattere pragmatico e per la sua attenzione per gli assetti costituzionali. Obiettivo centrale di questo convegno è mettere a fuoco la relazione fra teoria e politiche riformatrici in campo economico, giuridico e amministrativo. In questo ambito l'Illuminismo Lombardo è all'origine di due fra i più importanti contributi del Settecento riformatore italiano: la critica del sistema delle pene di Cesare Beccaria e le indagini di Pompeo Neri sui presupposti catastali della tassazione fondiaria. In entrambi i casi, un forte e originale impulso intellettuale è seguito da riforme istituzionali sia in Lombardia sia altrove (la Toscana e Parma sono i primi stati europei ad abolire la pena di morte, il nuovo sistema di tassazione della terra introdotto in Lombardia anche per effetto delle proposte di Neri esercita un'influenza profonda sulle riforme catastali in Inghilterra e Francia).

La prima parte di questo saggio si propone di mettere in risalto l'illuminismo delle riforme civili come specifica espressione di un atteggiamento riformatore radicato nella comprensione delle capacità economiche e istituzionali "interne" a specifici contesti. In questa prospettiva l'illuminismo degli economisti lombardi si distingue sia dall'illumi- 
nismo radicale dei Fisiocrati e degli economisti napoletani sia dall'illuminismo commerciale degli scrittori scozzesi di economia politica. La seconda parte del saggio discute il contributo degli illuministi lombardi allo studio dei processi di formazione della ricchezza, laddove quest'ultima è collegata ai flussi di reddito che si generano all'interno di un sistema di coordinamento fra attività distinte e specializzate (divisione del lavoro). Commercio e moneta sono considerati strumenti per la realizzazione concreta della divisione del lavoro e risultano in questo modo strettamente connessi a struttura e dinamica dei processi di produzione. La sezione successiva discute alcuni aspetti del contributo degli illuministi lombardi allo studio delle strutture normative e delle tecniche di amministrazione coerenti con sistemi efficaci di divisione del lavoro e formazione della ricchezza. Infine la sezione conclusiva considera la tematica complessiva delle relazioni fra politica ed economia negli scritti attraverso i quali si esprime l'illuminismo delle riforme civili.

\section{ECONOMIA POLITICA DELL'ILLUMINISMO CIVILE}

L'illuminismo degli economisti è un fenomeno complesso di cui è importante non trascurare la pluralità di articolazioni. Non diversamente da quello che accade per l'lluminismo in generale, anche l'illuminismo degli economisti si esprime attraverso analisi e proposte spesso molto lontane fra loro, anche se non è difficile individuarne una matrice comune. Ad esempio, la disposizione di coloro che, come i Fisiocrati, assumono il punto di vista dei "consiglieri esterni" rispetto all'autorità sovrana è profondamente diversa dalla prospettiva degli economisti che adottano l'ottica dei "commercianti colti" e sottolineano l'autonomia relativa di divisione del lavoro e mercati rispetto agli stati; così come è diversa dalla prospettiva di quegli economisti che si collocano per così dire in una posizione intermedia fra mercati e strutture di governo e ne esplorano le interdipendenze sul piano analitico e su quello dell'azione politico-amministrativa.

La riflessione economica degli illuministi lombardi si caratterizza per la capacità di visualizzare, all'interno di uno stesso sistema di analisi e di proposte, sia il punto di vista "orizzontale" della divisione del lavoro e degli scambi sia la prospettiva "verticale" degli apparati amministrativi. Si delinea in questo modo uno schema di economia politica e di governo dell'economia che è lontano dall'illuminismo 
radicale di Condorcet, Morellet, Paine, così come è lontano dal punto di vista del laisser faire "dirigista" di Quesnay e della sua scuola, e dal riformismo interventista di Genovesi e Tanucci. Il duplice inserimento di molti dei suoi esponenti (e in primo luogo di Cesare Beccaria e Pietro Verri) all'interno della classe dirigente milanese ma anche nelle strutture di governo dell'autorità centrale rende il loro contributo di particolare interesse. L'obiettivo principale di questa relazione è discutere il contributo degli illuministi lombardi alla definizione di un corpus di economia politica caratterizzato dalla sovrapposizione fra punto di vista della società commerciale e punto di vista degli apparati di governo (sovrapposizione che in questo convegno abbiamo inteso caratterizzare con l'espressione di illuminismo civile). I risultati di questo intreccio sono spesso di grande interesse per quanto riguarda la comprensione dei nessi tra divisione del lavoro e mercati, ordinamento giuridico e governo dell'economia. Come si è prima osservato, divisione del lavoro e mercati costituiscono ambiti privilegiati di attenzione per economisti che assegnano alle capacità di iniziativa e promozione di individui e gruppi un'efficacia autonoma rispetto alle decisioni politiche e alle loro conseguenze sul piano amministrativo. Questo spiega la centralità rispettiva di divisione del lavoro e commerci negli scritti economici di Beccaria e Verri. In particolare, il punto di vista dell'illuminismo civile suggerisce immediatamente la necessità di inserire la divisione del lavoro in un tessuto complesso di organizzazione "gerarchica" delle strutture produttive (Beccaria); lo stesso punto di vista indica l'esigenza di studiare commerci e mercati mettendo in risalto architetture istituzionali e configurazioni amministrative piuttosto che situazioni e criteri di scelta derivati per via inferenziale da principi di razionalità astratta (Verri).

In questa prospettiva, assume un ruolo centrale l'analisi delle azioni di governo intese come interventi "interni" alle stesse strutture della divisione del lavoro e dei mercati, e proprio per questa ragione efficaci a sollecitare e orientare capacità già esistenti all'interno del tessuto sociale. Diritto, amministrazione e politica monetaria configurano un quadro di analisi e di proposte che colloca al centro dell'interesse $\mathrm{i}$ processi di formazione della ricchezza e li considera parte integrante della struttura sociale nelle sue articolazioni istituzionali e amministrative. Si delinea in questo modo un insieme di implicazioni reciproche fra economia, politica e società che costituisce l'aspetto più caratteristico dell'illuminismo civile. 


\section{Divisione DEL LAVORO, COMMERCIO E CONFIGURAZIONI PRODUTTIVE}

Il periodo formativo dell'illuminismo economico lombardo si colloca attorno agli anni sessanta del '700 e riflette direttamente problemi e discussioni sul governo dell'economia nello Stato di Milano. Il nucleo centrale di queste riflessioni è costituito dalle condizioni della bilancia commerciale e dai collegamenti di queste con la gestione della politica monetaria. Un loro aspetto saliente è il rimando delle discussioni di politica commerciale e monetaria a schemi concettuali di carattere generale sui fondamenti delle azioni economiche e sui criteri guida delle interdipendenze fra le azioni di soggetti o gruppi (si veda si questo punto Quadrio Curzio e Scazzieri, 1986, pp. 29-38). A questo ambito di riflessioni appartengono alcuni scritti di Pietro Verri (Dialogo sul disordine delle monete nello Stato di Milano nel 1762, 1762; Considerazioni sul commercio dello Stato di Milano, 1761-1763), e lo scritto di Cesare Beccaria Del disordine e dè rimedj delle monete nello Stato di Milano nell'anno 1762, 1762. Aspetto comune di questi scritti è l'attenzione per il governo delle relazioni economiche inteso come combinazione di interventi attivi e di azioni volte a rimuovere ostacoli. In entrambi i casi, obiettivo centrale della politica economica è attivare disposizioni presenti nel contesto sociale piuttosto che realizzare interventi volti ad accrescere direttamente la felicità pubblica. Nel caso della politica commerciale, questo avviene attraverso un insieme coerente di misure protettive delle manifatture locali e al contempo di misure permissive per quanto riguarda i movimenti delle persone. Nel caso della politica monetaria, sono criticati i tentativi di assicurare stabilità attraverso la fissazione arbitraria del rapporto fra divise monetarie in circolazione, inefficace nello Stato di Milano 'essendo la sfera dei nostri contratti assai più vasta di quella dè confinì (Verri, 1986 [1772]), p. 127). In entrambi i contesti, il criterio guida è individuare soluzioni sulla base di un'accurata individuazione delle interdipendenze più rilevanti, evitando interventi a prima vista efficaci ma in realtà incapaci di incidere sulle origini dei problemi. Questi criteri generali sono precisati negli scritti successivi di Beccaria e Verri, in cui si delinea un quadro sistematico di principi di economia politica. La teoria delle connessioni di Verri è il principale presupposto di questa costruzione analitica. Come Verri osserva nelle Meditazioni sull'economia politica: 
'quanto l'uomo è più isolato e distante dagli altri suoi simili, tanto più si accosta allo stato selvaggio; all'opposto tanto più si accosta allo stato dell'industria e della coltura, quanto è più vicino a un gran numero di uomini; e deve farsi ogni studio possibile per accostare l'uomo all'uomo, il villaggio al villaggio, la città alla città' (Verri, 2007 [1771], pp. 505-506).

La struttura analitica che Verri deriva da questo punto di vista è articolata e profondamente originale: la dinamica delle connessioni deriva da una particolare configurazione dei bisogni e delle risorse per soddisfarli, e determina a sua volta cambiamenti strutturali nei processi di formazione e distribuzione della ricchezza.

Il punto di partenza dell'evoluzione parallela (ma non necessariamente sincronica) di bisogni e risorse è 'l'eccesso dei bisogni sopra il potere' (Verri, 2007 [1771], p. 395). Infatti, 'i selvaggi sono poco infelici perché hanno pochissimi bisogni; ma le nazioni che ne hanno acquistati in gran numero coll'incivilirsi, debbono di necessità cercare l'accrescimento della potenza per accostarsi alla felicità' (Verri, 2007 [1771], p. 396). Già a questo punto si individua una divaricazione fra sentieri alternativi di dinamica della ricchezza:

'Il bisogno spinge l'uomo talvolta alla rapina, talvolta al commercio. Perché vi sia commercio vi debbon essere bisogno e abbondanza: bisogno della merce che si cerca, abbondanza della merce che si cede in contraccambio. A misura che i bisogni crescono, cresce lo stimolo di aumentare le merci atte a cedersi in contraccambio' (Verri, 2007 [1771], p. 396).

La proposizione di Verri suggerisce la possibilità storica di molteplici punti di svolta: non sembra infatti che sia propria di Verri quella che si usa denominare interpretazione Whig della storia (Butterfield, 1931). ${ }^{1}$ A seconda della relazione tra bisogno e abbondanza, una società si trova a seguire traiettorie molto diverse l'una dall'altra. Bisogni in eccesso rispetto all'abbondanza possono spingere, in alternativa, alla rapina oppure al commercio a seconda di condizioni particolari che riguardano la dimensione della scarsità. Verri suggerisce che condizioni di scarsità relativa (piuttosto che scarsità assoluta) possano determinare il passaggio

1 Questa interpretazione tende a mettere in evidenza caratteri di evoluzione unilineare (progresso) nel passaggio da una fase storica all'altra, mettendo in secondo piano punti di arresto e processi involutivi. 
dalla rapina al commercio. Tuttavia il suo punto di vista porta ad escludere che questa svolta sia irreversibile: bisogni e abbondanza non sono necessariamente coordinati fra loro, per cui situazioni di momentanea scarsità assoluta possono condurre e regressi nella dinamica delle strutture sociali e nell'evoluzione della ricchezza:

'Nello stato di società i desiderii sono infiniti, perché nascono dalla fecondissima opinione sovrana degli uomini sociali, e il potere si accresce dal canto dell'industria e si scema da quello delle forze fisiche; ma se in questa società spira la barbara diffidenza, se l'esistenza e la proprietà diventano precarie, se dalla fonte dell'equità e della giustizia sgorga il terrore e la devastazione, il potere di ogni uomo è vacillante, e l'eccesso dè desiderii diventa sommo' (Verri, 1964 [1763], p. 120).

A questo punto la dinamica delle connessioni assume importanza centrale nel determinare la possibilità, anche se non la certezza, che i progressi della ricchezza abbiano luogo secondo il percorso del progresso civile. Infatti, secondo Verri,

'tanto più si estende la comunicazione, tanto più si moltiplicano le idee, tanto più si accrescono i bisogni, tanto cresce il commercio e parallela cresce l'agricoltura'(Verri, 2007 [1771], p. 401).

In questo modo, attraverso la moltiplicazione dei bisogni, diviene possibile generare situazioni di scarsità relativa e quindi dare avvio a nuove relazioni di scambio secondo la logica di un processo cumulativo. Il punto di svolta, come abbiamo visto, è il passaggio dalla scarsità assoluta alla scarsità relativa. La prima tende a facilitare la rapina anziché il commercio, la seconda rende possibile il commercio attraverso la moltiplicazione delle "riserve" di abbondanza relativa nel sistema economico. Come si è visto, lo schema analitico di Verri suggerisce che i progressi della ricchezza non abbiano carattere unidirezionale (non possono escludersi, anche in condizioni civili, situazioni di regresso determinate dal presentarsi di un eccesso generalizzato dei bisogni rispetto alle risorse disponibili). Tuttavia Verri sottolinea il carattere 'infelicissimo' di questi regressi e l'opportunità di evitarli senza essere costretti ad un drastico ridimensionamento dei bisogni:

'nessun altro partito resta da prendersi per le società già formate, se non quello di portarsi alla perfezione ed al massimo incivilimento con ottime 
leggi, ottimi costumi, e con ogni genere di coltura, addestrando la ragione e l'industria, ed affrettando i progressi della verità, fugando le opinioni a lei contrarie, e rendendo comune l'uso di essa à cittadini in quante azioni della vita si può' (Verri, 1964 [1763], p. 120).

In ultima analisi, secondo Verri, la dinamica delle influenze reciproche tra bisogni e risorse determina un campo di evoluzione strutturale in cui l'eccesso dei bisogni rispetto alle risorse può in alcuni casi dare avvio all'espansione delle connessioni attraverso il commercio (in altri casi, come Verri riconosce, la strada è aperta a conflitti armati e "rapina"). Conseguenza di questo è l'ulteriore moltiplicazione dei bisogni, come anche delle possibilità di soddisfarli attraverso divisione del lavoro e commerci su scala sempre più estesa. In altri termini, la dinamica dei bisogni e quella delle risorse sono in stretta dipendenza reciproca una volta che i sistemi economici abbiano oltrepassato la soglia della scarsità assoluta e dato avvio ai processi di differenziazione culturale e sociale che sono all'origine delle scarsità relative e dei mezzi per superarle tramite lo scambio. Centrale è quindi in Verri l'idea che incivilimento e commercio possano essere reciprocamente connessi secondo un percorso virtuoso che porta dalle scarsità relative alla divisione del lavoro, da quest'ultima all'ulteriore differenziazione dei bisogni, e dai nuovi bisogni a ulteriori approfondimenti di divisione del lavoro e relazioni di scambio. Come abbiamo visto, questo processo non ha nulla di necessario. In ogni momento, il circolo virtuoso della causalità cumulativa tra espansione delle risorse e differenziazione dei bisogni può arrestarsi, e fare luogo al circolo vizioso di un diverso processo cumulativo, quella tra contrazione delle risorse e progressivo appiattimento dei bisogni.

$\mathrm{Al}$ centro dello schema concettuale di Verri è la consapevolezza che le relazioni fra dinamica dei bisogni e dinamica delle risorse possano generare percorsi alternativi di cambiamento strutturale. I progressi della ragione sono strettamente collegati ai progressi della ricchezza se un sistema economico riesce ad avviare i rendimenti crescenti resi possibili dalla relazione tra differenziazione dei bisogni e sempre maggiore divisione del lavoro, e realizzati in concreto dall'espansione delle relazioni commerciali. ${ }^{2}$ D'altra parte, condizioni di

2 Come ha osservato Angelo Moioli, "[V]erri si era convinto che era il commercio stesso, inteso come fenomeno dinamico, a generare la ricchezza: in quanto tale, 
degrado civile accompagnano le vicende di un sistema economico in cui risulti bloccato "il moto dell'industria" (Verri, 2007 [1771], p. 457). In questo caso può avviarsi un processo cumulativo di rendimenti decrescenti in cui la contrazione nelle capacità di risposta del sistema produttivo induce un progressivo restringimento sia degli scambi sia dei bisogni: "accaderà che fra uomo e uomo la contrattazione si riduca e limiti al minor grado, e proporzionatamente si diminuirà la riproduzione annua; e la Nazione povera, isolata e languente ripiegherà verso gli antichi suoi principj, allontanandosi dallo stato della coltura" (Verri, 2007 [1771], p. 456).

La maggiore o minore corrispondenza tra bisogni e risorse è anche il punto di partenza delle riflessioni di Cesare Beccaria sul processo di incivilimento, di cui i progressi della ricchezza sono considerati componente essenziale:

'La barbarie di una nazione, se si prenda in un senso preciso e filosofico, non è altro che la ignoranza delle cose utili a quella, e dei mezzi più pronti e più conformi alla felicità particolare di ciascheduno per ottenerla; la coltura di una nazione è la cognizione di tutto ciò ... Finchè le cognizioni e le opinioni sono in equilibrio coi bisogni e colla massima felicità conosciuta di ciascheduno in particolare, non può chiamarsi barbara una nazione, ma può essere più o meno selvaggia, termine che esprime la maggior o minore lontananza dalla massima unione che possa darsi fra gli uomini, e dalla massima assoluta felicità possibile divisa nel maggior numero possibile ... Una nazione può essere selvaggia e barbara; può essere selvaggia e non barbara; può essere barbara e molto socievole nel medesimo tempo' (Beccaria, 1971 a [ms. circa 1767-1769], p. 802).

In Beccaria, principi di associazione e divisione spiegano sia la dinamica della conoscenza sociale sia il progresso dei mezzi materiali per soddisfare interessi e bisogni. Nei Pensieri sopra le usanze e $i$ costumi (Beccaria, 1971 b [ms. circa 1767-1769], si prende in esame la relazione fra struttura delle disposizioni abituali (usanze) e coesione sociale:

'La molteplicità delle usanze, moltiplicando le sensazioni e le occupazioni, diminuisce la forza delle passioni, amansa le nazioni feroci, ma inde-

egli lo aveva posto al centro della sua proposta, attribuendo ed esso una forza propulsiva in grado, se non di fare 'riascendere al colmo dell'antica prosperità' l'economia locale, di avviare comunque un significativo percorso di 'accrescimento delle ricchezze" (Moioli, 2006, p. 48). 
bolisce le mansuete; aumenta lo spirito di società esterna per cui i corpi si avvicinano, ma gli animi s'allontanano. Perché i grandi interessi, nei quali si può senza grave discapito far qualche sagrificio per combinarsi, riuniscono gli uomini; ma i piccioli, che col cadere si annullerebbero, li dividono: ora gli usi essendo mezzi per ottenere i piaceri della società, moltiplicandosi, suddividono in picciole parti gli interessi, ed allontanano gli animi' (Beccaria, 1971 b [ms. circa 1767-1769], p. 814).

La relazione fra bisogni (interessi) e mezzi per soddisfarli acquista una nuova dimensione passando alla dinamica degli uni e degli altri attraverso processi di differenziazione sociale. In questo caso, Beccaria individua con chiarezza la corrispondenza fra distinzione degli interessi e "reciproci aiuti":

'in tutti i tempi e in tutte le condizioni essendo avvezzi gli uomini dalle scambievoli relazioni e dai reciproci aiuti, non solamente a soddisfare i bisogni, ma eziandio a soddisfarli piacevolmente e comodamente; e ciascuno osservando che tanto più piacevolmente e comodamente vivea quanto maggior numero dè suoi simili potea indurre a procurargli questi comodi e piaceri, e che ciò più facilmente e più frequentemente otteneva, quanto più sopra gli altri poteva rendersi osservabile e distinto; nacque negli uomini il bisogno dei piaceri, ossia l'indeterminato sentimento di privazione, ossia la noia e la voglia di distinguersi' (Beccaria, 1971 c [ms. 1769], p. 615).

Analogamente a Verri, Beccaria sottolinea che la differenziazione di bisogni e risorse è all'origine dei progressi della ricchezza. Infatti la distinzione fra bisogni assegna carattere relativo ad abbondanza e scarsità, così che 'le abbondanze si compensino colle scarsezze, e mettansi le une colle altre al dovuto livello' (Beccaria, 1971 c [ms. 1769], p. 445). In ultima analisi, i progressi della ricchezza sono attribuiti a processi di separazione che partendo dalla differenziazione delle attività produttive attraverso la divisione del lavoro (le "scambievoli relazioni" e i "reciproci aiuti") rendono più facile il soddisfacimento dei bisogni e ne incoraggiano il progressivo affinamento. In questa prospettiva, la comunicazione reciproca fra soggetti o gruppi può realizzarsi attraverso il commercio, ma è generata da dinamiche strutturali che riguardano in primo luogo l'evoluzione delle conoscenze e le interdipendenze oggettive fra processi di produzione. L'attenta considerazione degli aspetti materiali delle strutture sociali è uno degli aspetti più caratteristici del punto di vista di Beccaria circa il processo di incivilimento e i progressi delle ricchezze. Questo punto di vista è il risultato di una complessa linea di 
ragionamento che è opportuno esaminare brevemente. Già nel saggio Del disordine e dè rimedj delle monete nello Stato di Milano nell'anno 1762 (Beccaria, 1986 [1762]) Beccaria aveva espresso un atteggiamento critico nei confronti del contrattualismo nella teoria sociale e nella filosofia giuridica e politica:

'Lintroduzione delle monete non nacque da una espressa convenzione (la quale non ha preceduto mai veruno universale stabilimento), ma bensì da ciò che il volgo chiama azzardo, cioè da una disposizione di circostanze non premeditata dagli uomini' (Beccaria, 1986 [1762], p. 196).

Nelle successive Ricerche intorno alla natura dello stile (Beccaria, 1971 d [1770], Beccaria conferma e precisa questo punto di vista in una discussione sull' 'origine della giustizia e dell'idea che di quella si formano gli uomini' (Beccaria, 1971 d [1770], p. 332):

'Figuriamoci che si tratti di ricercare l'origine della giustizia e dell'idea che di quella si formano gli uomini. Finchè noi ci fermeremo a combinare la parole astratte, e i termini generali che sono relativi alla parola giustizia, alle quali non corrispondono che pochissime e sfuggevolissime idee nella mente nostra, noi arriveremo forse a tessere una lunga diceria la quale annoierà gli ascoltanti e gli renderà disattenti e disgustati dal non sentire calmata l'inquietudine della curiosità, e del non trovarsi eccitate dal discorso e dalla lunga serie di suoni idee chiare e determinate nella mente loro. Finchè noi, per esempio, diremo che la idea della giustizia è nata negli uomini dal bisogno che hanno avuto di conservare la pace e la tranquillità delle famiglie; ... finchè diremo consistere questa in una esatta proporzione e distribuzione delle cose, a misura dè diritti acquistati da ognuno, e questi diritti non essere altro per noi che quanto ci detta la ragione essere conforme alla umana natura; finchè noi queste e simili cose diremo, noi avremo detto una serie di parole tutte corrispondentisi tra di loro e tutte derivanti da certe ed inconcusse verità; ma pochissime idee saranno ciò non ostante risvegliate nella mente di chi ascolta; l'idea complessa della giustizia non sarà chiaramente determinata, non saremo né convinti né appagati delle cose dette, ed il dubbio e la confusione resteranno nella mente' (Beccaria, 1971 d [1770], pp. 332-333).

Nel passo che precede è evidente la critica dei tentativi di spiegare gli assetti istituzionali in termini contrattualistici alla Hobbes, così come l'esigenza di trovare una spiegazione alternativa nelle concrete situazioni che "risvegliano" idee complesse al di là delle "parole astratte". Centrali in questa prospettiva sono le condizioni di fatto che determinano la posizione delle persone e dei gruppi sociali, e che finiscono per dare origine a particolari cognizioni e costumi: 
'[S]e invece chi ascolta o chi medita per esporre chiaramente ciò di cui si tratta, avrà cura di fissare la sua attenzione alle poche idee sensibili e particolari che si risvegliano in quest'occasione, nella quale si ricerca l'origine della giustizia, come uomini, famiglie, sostentamento, fatica ec., egli si sforzerà di chiaramente imaginarsi tali idee e farsele sensibili, e non perdendole giammai di mira, di trovare ciò che hanno di comune con esse le parole generali pace, tranquillità, incertezza, sicurezza, diritto, umana natura ec. Per ciò fare, egli si rappresenterà a se medesimo se stesso od altri individui, come aventi necessariamente fame, sete, bisogno di coprirsi e di difendersi dalla crudeltà delle stagioni e delle fiere, onde essere una conseguenza necessaria della propria organizzazione il cercar e prendere quelle cose che sono più abili a dissetarlo, sfamarlo, coprirlo e difendere, e ciò più prontamente e più sicuramente, e di tralasciar quelle ricerche che rade volte lo soddisfacciano, ed a costo di molti pericoli e dolori' (Beccaria, 1971 d [1770], p. 333, corsivo aggiunto).

La prospettiva di Beccaria individua un nesso strettissimo fra condizioni di fatto e organizzazione delle azioni umane. Per questa ragione l'ipotesi contrattualistica della "espressa convenzione" appare lontana dalla realtà e le strutture oggettive passano in primo piano nella spiegazione dei fenomeni sociali. ${ }^{3}$ L'interesse di Beccaria per le condizioni che permettono di conseguire il risultato della massima felicità pubblica, così come la sua attenzione per gli ostacoli che debbono essere rimossi in vista di quell'obiettivo, sono alla base di analisi economiche che pongono al centro configurazioni alternative dei diritti di proprietà e interdipendenze oggettive fra processi di produzione. Il complesso di questi ragionamenti finisce per "cristallizzarsi ....in una teoria dell'economia politica” (Venturi, 1997 (1958), p. 17): "Lo sviluppo dell'umanità, che sembrava abbandonato all'arbitrio delle ipotesi quando s'intendeva ricostruirlo partendo dalle idee di 'barbarie' o di 'ripulimento', sembrava diventare comprensibile e misurabile quando si trattava dell'accrescersi della ricchezza delle nazioni. L' 'economia pubblica' era lo sbocco e in qualche modo la conclusione della ricerca di Beccaria" (Venturi, 1997 [1958], p. 18; si veda anche Audegean, 2010).

Le riflessioni economiche di Beccaria sono guidate dalla ricerca di un criterio uniforme di sistemazione concettuale per campi di analisi a

3 In un passo successivo delle Ricerche intorno alla natura dello stile Beccaria afferma con ancora maggiore forza questo punto di vista: "[n]oi troveremo [...] che il sentimento di giustizia nacque coll'umana società, che i soli rapporti fisici degli uomini tra di loro potevano produrlo e conservarlo" (Beccaria, 1971 d [1770], p. 335; corsivo aggiunto). 
prima vista distanti come agricoltura e manifatture, commercio e tributi (Beccaria, 1971 e [ms. 1768], p. 342):

"è necessario rimontare all'origine delle cose stesse, ove solo si può rinvenire qualche primitiva e primaria combinazione, che è stata come il nucleo o punto di appoggio, intorno al quale si sono raggruppati ed avvolti i molteplici e diversi dettagli d'una scienza" (Beccaria, 1971 c [ms.1769], p. 385).

E questo "nucleo" è individuato da Beccaria in due proposizioni o "principi" fondamentali:

"Dunque, raccogliendo le cose fin qui dette, primo principio d'ogni operazione economica sarà quello d'eccitare la maggior quantità possibile di prodotto utile e contrattabile, e di togliere di mezzo ciò che diminuisce questa massima quantità di prodotti [...] [I]l secondo principio d'ogni economica operazione, riguardo alle opere della mano e dell'industria, sarà quello di fare piccoli per volta, ma più spessi guadagni che sia possibile" (Beccaria, 1971 c [ms. circa 1769], p. 390).

I due principi risultano coordinati fra loro: il primo riguarda la dinamica delle capacità produttive e la realizzazione della massima quantità possibile di prodotto netto in condizioni date; il secondo fa riferimento alla dinamica di divisione del lavoro, estensione dei mercati e domanda effettiva. In entrambi i casi, è centrale il presupposto della riproduzione del prodotto complessivo, e quindi la ricostituzione delle "scorte annue $[\ldots]$ necessarie a continuare la riproduzione" (Beccaria, 1971 c [ms. circa 1769], p. 387). Tuttavia le condizioni della riproduzione sono diverse a seconda dei settori produttivi, e di conseguenza a seconda dei sistemi economici in cui quei settori possono essere rispettivamente principali o secondari:

"Il primo principio è il reggitore dell'economia agricola e fondamentale di una nazione; il secondo è il principio dell'economia artista ed industriosa della medesima. Al primo debbono principalmente applicarsi le nazioni che hanno un territorio, ed avere il secondo principio subalterno e secondario; a questo, quelle che prive sono di un territorio fertile e riproducente. Ma tali nazioni che campano sulla sola industria ed opera delle loro mani non esisterebbero, se non esistessero terre feconde da nazioni agricole lavorate" (Beccaria, 1971 c [ms. circa 1769], pp. 390-391).

Lo schema concettuale dell'economia come flusso circolare di beni 
prodotti e riprodotti è alla base delle analisi economiche di Beccaria e delle sue proposte di politica economica. In questa prospettiva è evidente l'influsso della letteratura fisiocratica francese, benché sia caratteristico di Beccaria il tentativo di individuare, all'interno del sistema economico, una pluralità di 'sub-sistemi circolari' fra loro relativamente indipendenti e quindi capaci di determinare condizioni di riproduzione ed espansione diverse da un sub-sistema all'altro. Questo punto di vista consente di mettere in evidenza sia le interdipendenze fra processi produttivi all'interno di un'economia circolare sia le posizioni relative dei diversi sub-sistemi all'interno di una configurazione gerarchica di processi. In particolare, Beccaria riconosce la centralità delle materie prime in ciascun sistema economico o insieme di sistemi economici fra loro integrati (vedi sopra). ${ }^{4} \mathrm{Al}$ tempo stesso, si attribuisce ai processi di trasformazione delle materie prime un ruolo determinante nell'individuare la posizione di ciascun sub-sistema all'interno del sistema economico, così come la posizione di ciascun sistema economico (o "nazione") all'interno di un insieme integrato di tali sistemi. Questo criterio individua una precisa proporzione fra livelli di occupazione nei diversi settori produttivi:

"Le classi delle persone che travagliano debbono essere tanto meno popolate, quanto maggiore è la distanza dalle classi immediatamente alimentatrici ... [N]oi troveremo sempre la classe producente l'alimento la più numerosa, e la classe lavoratrice la meno numerosa in proporzione della distanza sua dalla produzione immediata dell'alimento medesimo" (Beccaria, 1971 c [ms. circa 1769], pp. 395-396).

In conclusione, lo schema di riproduzione di Beccaria illustra, a partire da condizioni necessarie di interdipendenza oggettiva fra settori, l'esistenza di possibilità alternative per quanto riguarda le modalità concrete di organizzazione dei sistemi economici. Ad esempio, secondo

4 Beccaria osserva che "i confini politici d'uno stato non [sono] sempre o quasi mai gli stessi dè confini economici di quello" (Beccaria, 1971 c [ms. circa 1769], p. 391). Una conseguenza importante è che: "[l]a terra di una nazione alimenta l'industria di un'altra, l'industria di questa feconda la terra di quella: queste due nazioni, quantunque divise di sovranità ed indipendenti reciprocamente dalle rispettive loro leggi politiche, sono però realmente una sola nazione strettamente unita per leggi fisiche, e dipendenti l'una dall'altra per le relazioni economiche" (Beccaria, 1971 c [ms. circa 1769], p. 391). 
Beccaria, il criterio di proporzionalità sopra descritto potrebbe non essere soddisfatto in particolari sistemi economici. Tuttavia lo stesso criterio risulta soddisfatto una volta che si considerino "le terre forastiere somministranti l'alimento rappresentatore del soverchio travaglio [lavoro in sovrappiù] di una nazione, come facenti realmente un corpo solo colle terre alimentatrici della nazione medesima" (Beccaria, $1971 \mathrm{c}$ [ms. circa 1769], pp. 395-396). Diversi criteri di interdipendenza fra "nazioni" producono quindi effetti molto diversi a seconda del peso relativo dei diversi settori produttivi nelle "nazioni" fra loro connesse. Infatti risulteranno avvantaggiate nella formazione e distribuzione del prodotto complessivo quelle "nazioni" in cui le proporzioni fra settori rispettano l'ordine di priorità determinato dalla produzione e trasformazione di materie prime:

"Il soverchio [l'eccedenza] dunque del travaglio sopra del bisogno della propria nazione le sarà tanto più utile quanto questo soverchio sarà nelle classi sempre più vicine a rappresentar la propria proporzionata quantità di alimento. Primo, perché essendo le più numerose, la distribuzione degli utili cadrà sopra un maggior numero di persone, il che servirà ad [incoraggiare] più immediatamente i lavori più necessari e più utili. Secondo, perché l'accrescimento delle arti inferiori, cioè più vicine alla produzione dell'alimento, anima le superiori; ma l'accrescimento di queste non anima egualmente quelle. La superiorità dè loro guadagni mettendole nel caso di supplire fuori di stato alla più parte dei bisogni, le classi loro intermedie possono essere forastiere piuttosto che nazionali, ed essere più utili all'altrui che alla propria nazione" (Beccaria, 1971 c [ms. circa 1769], p. 396).

La rappresentazione analitica del sistema economico utilizzata da Beccaria mette in evidenza interdipendenze fra componenti del sistema diverse dalle connessioni fra soggetti o gruppi considerate da Verri. Questo fa sì che sia le opportunità sia i vincoli di fronte ai quali si trovano i processi di formazione di ricchezza siano visti sotto una luce diversa. Ad esempio caratteristica di Beccaria è l'attenzione per "il principio di complementarietà tra l'agricoltura e l'industria [...] e [...] l'idea che lo sviluppo delle manifatture possa contribuire a sostenere una popolazione maggiore di quanto permetterebbe la terra del paese" (Porta, 1990, p. 364)..$^{5}$ Al tempo stesso Beccaria è consapevole che non

5 È interessante rilevare che il "principio di complementarietà" sviluppato da Beccaria nella sua analisi delle condizioni strutturali della dinamica economica richiede 
tutte le tipologie di sviluppo delle manifatture sono egualmente atte ad assicurare una distribuzione soddisfacente degli incrementi di ricchezza fra i settori di un sistema economico. Infatti una dinamica della ricchezza guidata dalle esportazioni di manufatti di lusso può risultare vantaggiosa per gruppi sociali ristretti nel paese esportatore, ma ancora di più (data la catena di effetti indiretti) per i settori che producono all'estero le corrispondenti materie prime. Infatti la crescita delle "arti superiori" non genera adeguati incrementi di domanda effettiva interna per i beni ad esse necessari (le rispettive "classi intermedie"), e le rende a loro volta dipendenti dai mercati internazionali per quanto riguarda la fornitura di prodotti intermedi.

\section{SCHEMI DI INTERDIPENDENZA E GOVERNO DELL'ECONOMIA}

Le riflessioni di Pietro Verri e Cesare Beccaria sui progressi della ricchezza presentano importanti punti di contatto ma anche rilevanti elementi di differenziazione. Entrambi pongono al centro delle rispettive analisi il processo di incivilimento, considerato nei termini dell'interazione fra dinamica dei bisogni e dinamica delle risorse atte a soddisfarli. Ma già a questo proposito può essere rilevata un'importante differenza. Per Verri gli scambi commerciali e le comunicazioni reciproche ad essi collegate sono all'origine di un processo in cui le scarsità da assolute divengono relative e i bisogni tendono a moltiplicarsi (vedi sopra). Una conseguenza importante della moltiplicazione dei bisogni è la crescente interdipendenza fra soggetti e/o gruppi attraverso la divisione del lavoro, e quindi il progressivo allontanamento dalle condizioni di scarsità assoluta che spingono alla "rapina" piuttosto che al "commercio". Per Beccaria, invece, la divisione del lavoro precede lo scambio, nel senso che le connessioni originarie fra individui e/o gruppi sono spiegate attraverso condizioni di fatto che precedono specifiche modalità di interazione (quali quelle che possono realizzarsi attraverso il

di utilizzare sia il criterio dell'integrazione orizzontale fra processi interdipendenti all'interno di una data economia circolare di produzione e consumo sia il criterio dell'integrazione verticale fra stadi successivi di uno stesso processo o insieme di processi (si vedano Pasinetti, 1973; Pasinetti, 1981; Baranzini e Scazzieri, 1990; Quadrio Curzio e Scazzieri, 1990; Quadrio Curzio e Pellizzari, 1999). 
commercio). Questo punto di vista deriva dall'attenzione di Beccaria per i "rapporti fisici" fra gli uomini, e quindi per le "conseguenze necessarie" dei corrispondenti schemi organizzativi (vedi sopra).

Le premesse teoriche di Verri e Beccaria presentano rilevanti punti di contatto. Tuttavia lo studio dei rispettivi punti di vista mette in evidenza aspetti diversi del progresso della ricchezza e del governo dell'economia all'interno di un quadro concettuale solo a prima vista omogeneo. Alcuni esempi illustrano la prossimità e insieme la distanza tra Beccaria e Verri attorno a importanti questioni di politica economica. Il periodo iniziale della loro collaborazione intellettuale coincide con la controversia monetaria che si svolge a Milano a partire dal 1761-62. Lo scritto di Beccaria Del disordine e dè rimedj delle monete nello Stato di Milano nel'anno 1762 (Beccaria, 1986 [1762]) è seguito da scritti di Pietro Verri (Verri, 1986 a [1762], Verri 1986 b [1772]), in cui la posizione di Beccaria, contraria all'introduzione di una "moneta provinciale" nello Stato di Milano, viene difesa sulla base della duplice argomentazione che il disordine monetario riflette l'errore delle leggi civili qualora queste ultime siano in contrasto con le leggi economiche, e che sia opportuno lasciar battere moneta propria alle nazioni fornite di risorse naturali o di particolari monopoli marittimi (Verri, 1986 a [1762]). Tuttavia, già in queste discussioni, si avverte, nei due autori, un'enfasi diversa su questioni specifiche. Beccaria propone di fissare il rapporto tra monete in circolazione in base al corrispondente rapporto fra metalli monetati (oro e argento), e di regolare quest'ultimo in base al "comune livello" che si stabilisce fra le "nazioni diverse d'Europa", essendo queste "tanto internate reciprocamente per un incessante e vivo commercio" da potere considerarsi "come una sola nazione" (Beccaria, 1986 [1762], p. 59). Verri invece fa riferimento alla "opinione comune" in specifiche condizioni di tempo e luogo (Verri, 1986 b [1772]). La differenza nelle soluzioni tecniche proposte dai due economisti a partire dallo stesso schema di analisi monetaria è indicativa di differenze più profonde che verranno alla luce nei loro contributi successivi alla teoria e pratica dell'economia politica. Un interessante terreno di confronto è costituito dall'agricoltura e dal suo contributo alla ricchezza complessiva di un sistema economico. Secondo Verri, occorre partire dalla premessa che "[o]gni genere di agricoltura è utile allo Stato, perché accresce l'annua riproduzione: ma quel genere di agricoltura sarà preferibile, che più accresce l'annua riproduzione" (Verri, 2007 [1771], p. 507, corsivo dell'autore). A questa proposizione generale 
Verri aggiunge una serie di precisazioni. In particolare, Verri afferma che si debbano preferire attività che portino al livello più elevato di occupazione, che siano da evitare coltivazioni che potrebbero portare a degrado ambientale, che siano da sostenere produzioni di prima necessità in grado di assicurare autosufficienza alimentare (Verri, 2007 [1771], pp. 508-510). In tutti questi casi, tuttavia, Verri è fermo nel rigettare interventi diretti di carattere coercitivo:

"[Q]uando io dico che questi oggetti sono degni dell'attenzione del Legislatore, e che un genere merita d'essere più promosso, e un altro più ristretto, non intendo dire perciò, che io creda mai l'obbligare i proprietarj con leggi dirette o penali ad abbandonare o scegliere una coltura più che un'altra" (Verri, 2007 [1771], pp. 510-11).

Gli strumenti utili alla diffusione delle coltivazioni più adatte al progresso della ricchezza sono incentivi piuttosto che coercizioni, e gli stessi interventi realizzati con questo obiettivo debbono limitarsi ad assicurare la stabilità del processo di crescita della "annua riproduzione", senza mirare ad incidere più in profondità nella composizione dei processi produttivi. ${ }^{6}$ Il punto di vista di Beccaria è in apparenza simile ma deriva da una struttura analitica molto diversa. In particolare, il concetto di riproduzione in Beccaria è direttamente collegato alla considerazione del sistema economico come flusso circolare di produzione e consumo (vedi sopra). In questa prospettiva, Beccaria considera esplicitamente le interdipendenze fra processi agricoli e processi manifatturieri, così come le proporzioni fra attività produttive che da esse derivano:

"la quantità di terra impiegata nella produzione della materia prima d'una tal'arte sarà tanto minore, quanto maggiore sarà la distanza d'una tal'arte dall'immediata consumazione" (Beccaria, 1971 c [ms. circa 1769], p. 462).

Come Verri anche Beccaria ritiene che lo strumento operativo più adeguato per incoraggiare certe coltivazioni al posto di altre siano incentivi e aggravi tributari, ma i presupposti analitici sono diversi.

6 Verri osserva che "[u]n Ministro politico non sarà mai di altro sollecito; e ottenuto che siasi il necessario fisico non si curerà se sia variata o no la coltura; se molte materie prime delle arti si producono; se cresca sul suolo quanto serve ai comodi della vita; poiché ciò si livella da sé; [...] L'idea di formare un compendio dell'universo entro i proprj confini non è mai ben augurata" (Verri, 2007 [1771], p. 512). 
Infatti Beccaria presta grande attenzione all'introduzione di proporzioni tra processi produttivi che consentano di ottenere la maggior quantità possibile di "prodotto utile", data l'interdipendenza fra gli stessi processi in un dato sistema economico, o in un dato insieme di sistemi economici fra loro integrati. Verri invece evita di addentrarsi nelle studio delle interdipendenze oggettive fra processi e assegna all'obiettivo della massima riproduzione annua un significato macroeconomico. ${ }^{7} \mathrm{Le}$ distinte premesse analitiche fanno sì che gli stessi strumenti di politica economica (nel caso specifico, di politica tributaria) acquistino un significato diverso nei due economisti perché diversi sono i "punti sensibili" del sistema economico ai quali possono essere applicati.

Il problema dell'annona (e quindi le modalità operative volte ad evitare situazioni di scarsità e carestia negli approvvigionamenti di beni prima necessità) è "una delle grandi questioni teoriche e pratiche" nell'Italia di metà Settecento (Porta, 2007, p. 3). Questo tema, come osserva Pier Luigi Porta, "è all'intersezione di un nodo di problemi di economia politica e politica economica, che vanno [...] dal ruolo dell'agricoltura nello sviluppo economico, alla libertà di commercio, alla specie e applicazione dell'imposta" (Porta, 2007, p. 3). Anche su questo tema le posizioni di Verri e Beccaria mostrano punti di convergenza e linee di divaricazione che possono ricondursi alle differenze fra i rispettivi modelli fondamentali di rappresentazione del sistema economico. Infatti Verri accoglie senza riserve il punto di vista secondo cui problemi di approvvigionamento derivano quasi esclusivamente da privilegi e monopoli piuttosto che da condizioni di scarsità generalizzata (Verri, 2007 [1769]). ${ }^{8}$ Beccaria è invece più disposto ad esaminare criticamente le posizioni rispettive dei "partigiani della limitazione" e dei "partigiani della libertà". Infatti egli riconosce che "generalmente la libertà assoluta, ossia il non sistema, [sia] il migliore di tutti i sistemi che in materia di annona si possano immaginare" (Beccaria, 1971 c [ms. circa 1769], p.

7 Resta il fatto che per Beccaria come per Verri tributi mirati sono "uno dei più potenti strumenti di politica economica: se usati correttamente essi possono stimolare, mediante un'apposita struttura dei dazi, l'attività economica e in particolare il commercio e l'industria, ma possono, se applicati malamente, costituire ostacolo rilevante al buon funzionamento dell'economia" (Bognetti, 2007, pp. 653-654).

8 Pier Luigi Porta richiama l'affinità tra il punto di vista di Verri e la posizione di recente espressa da Jean Drèze e Amartya Sen circa il problema annonario nell'India contemporanea (Porta, 2007, p. 11). 
477). Tuttavia ammette che in particolari condizioni geografiche e istituzionali un sistema economico non possa trarre vantaggio dal funzionamento dei mercati e si trovi di fronte non tanto al maggiore prezzo della derrata ma alla "privazione istantanea" e "mancanza" di questa (Beccaria, 1971 c [ms. circa 1769], p. 477). In queste condizioni, secondo Beccaria, è essenziale "frenare l'interesse personale tra quei limiti nè quali divenga una forza combinata col bene pubblico, non una contraria e distruttiva di quello" (Beccaria, 1971 c [ms. circa 1769], p. 478).

\section{ECONOMIA E POLITICA DELLE RIFORME CIVILI}

Questo saggio si è concentrato sui contributi di Cesare Beccaria e Pietro Verri sia per il loro interesse intrinseco sia per la luce che tali contributi gettano sulle tensioni interne e sulle dinamiche di quello che abbiamo chiamato illuminismo delle riforme civili. Infatti i contributi dei due economisti alla teoria e alla pratica dell'economia politica sono indicativi sia della prospettiva comune sia delle divaricazioni che caratterizzano le analisi economiche condotte nello Stato di Milano attorno alla metà del diciottesimo secolo. Beccaria richiama l'attenzione sulle complementarietà fra processi produttivi, sulle gerarchie che si stabiliscono fra questi processi, e sulle conseguenze di queste forme di interdipendenza per quanto riguarda struttura complessiva e dinamica dei sistemi economici. Pietro Verri si concentra a sua volta sulla dinamica della ricchezza e collega quest'ultima alla migliore possibile utilizzazione delle capacità produttive di un sistema economico.

Al di là di questi punti di partenza, a prima vista prossimi, Beccaria e Verri finiscono per mettere in luce aspetti diversi, anche se spesso complementari, dei progressi della ricchezza. In Beccaria si rivelano centrali le interdipendenze oggettive costruite a partire da condizioni di fatto (piuttosto che da esplicite convenzioni) e radicate nei "rapporti fisici" fra esseri umani. Questo punto di vista lo porta quasi naturalmente a prestare attenzione ai vincoli strutturali (anch'essi "fisici" perché conseguenza della tecnologia in uso) che condizionano la dinamica dei sistemi produttivi (così come dei sub-sistemi che li compongono), e le loro reciproche dipendenze (spesso asimmetriche) sul piano interno e internazionale. In Verri, al contrario, acquista rilievo l'attenzione per le interdipendenze soggettive generate attraverso processi di comunicazione e consapevole deliberazione da parte di sogget- 
ti e gruppi sociali. Il punto di vista di Verri pone al centro i "rapporti morali" fra esseri umani e lo conduce naturalmente a porre enfasi sui vincoli istituzionali, e quindi sui collegamenti tra analisi delle disposizioni morali e scienza della legislazione.

La divaricazione appena descritta non è sempre in primo piano, eppure essa costituisce la matrice analitica dei diversi approcci seguiti da Beccaria e Verri nei rispettivi tentativi di dare unità concettuale alla nuova scienza dell'economia politica. Questa divaricazione è anche centrale per comprendere le ragioni delle differenze fra i due economisti attorno ai vincoli delle decisioni politiche in vista dell'obiettivo, condiviso da entrambi, di rendere massima la "riproduzione annua" di un sistema economico. 


\section{BIBLIOGRAFIA}

Audegean, P. (2010) La philosophie de Beccaria. Savoir punir, savoir écrire, savoir produire, Paris, Vrin, 2010.

Baranzini, M. e Scazzieri, R. (1990) 'Economic Structure: Analytical Perspectives', in M. Baranzini e R. Scazzieri, a cura di, The Economic Theory of Structure and Change, Cambridge, Cambridge University Press, pp. 227-333.

Beccaria, C. (1971 a; ms. 1767-69) 'Pensieri sopra la barbarie e coltura delle nazioni e su lo stato selvaggio dell'uomo', in C. Beccaria, Opere, a cura di S. Romagnoli, Firenze, Sansoni, vol. II, pp. 802-809.

Beccaria, C. (1971 b; ms. 1767-69) 'Pensieri sopra le usanze e i costumi', in C. Beccaria, Opere, a cura di S. Romagnoli, Firenze, Sansoni, vol. II, pp. 810-822.

Beccaria, C. (1971 c [ms. circa1769]) Elementi di economia pubblica, in C. Beccaria, Opere, a cura di S. Romagnoli, Firenze, Sansoni, vol. I, pp. 383-649.

Beccaria, C. (1971 d [1770]) Ricerche intorno alla natura dello stile, in C. Beccaria, Opere, a cura di S. Romagnoli, Firenze, Sansoni, vol. I, pp. 197-336.

Beccaria, C. (1971 e [1768]) 'Piano d'istruzioni per la cattedra di scienze camerali o sia di economia civile', in C. Beccaria, Opere, a cura di S. Romagnoli, Firenze, Sansoni, vol. I, pp. 341-349.

Beccaria, C. (1986 [1762]) Del disordine e de' rimedi delle monete nello stato di Milano nell'anno 1762, in Sul disordine nelle monete a Milano nel Settecento, tre saggi di Cesare Beccaria e Pietro Verri, introduzione di A. Quadrio Curzio e R. Scazzieri, quadri storici di M. Bortolotti e B. Cereghini, Milano, Electa per Banca del Monte di Milano.

Bognetti, G. (2006) 'Nota introduttiva' a 'Tributi, Monete e Dazi', in P. Verri, Scritti di economia, finanza e amministrazione, tomo II (Edizione nazionale delle opere di Pietro Verri, vol. II), Roma, Edizioni di storia e letteratura, pp. 651-691.

Butterfield, H. (1931) The Whig Interpretation of History, London, G. Bell and Sons.

Capra, C. (2002) I progressi della ragione. Vita di Pietro Verri, Bologna, Il Mulino.

Moioli, A. (2006) 'Nota introduttiva', in P. Verri, Scritti di economia, finanza e amministrazione, a cura di Giuseppe Bognetti, Angelo Moioli, Pierluigi Porta, Giovanna Tonelli, tomo I (Edizione nazionale delle opere di Pietro Verri, vol. II), Roma, Edizioni di storia e letteratura.

Mondolfo, R. (1925) 'Cesare Beccaria e l'opera sua', in C. Beccaria, Opere scelte, con introduzione e note di R. Mondolfo, Bologna, Cappelli, pp.v- xliv.

Pasinetti, L.L. (1973) 'The Notion of Vertical Integration in Economic Analysis', Metroeconomica, xxv, pp. 1-29.

Pasinetti, L.L. (1981) Structural Change and Economic Growth. A Theoretical Essay on the Dynamics of the Wealth of Nations, Cambridge, Cambridge University Press.

Pasinetti, L.L. (2007) Keynes and the Cambridge Keynesians. A 'Revolution in Economics' to be Accomplished, Cambridge, Cambridge University Press. 
Porta, P.L. (1990) 'Le lezioni di economia di Cesare Beccaria', in Cesare Beccaria tra Milano e l'Europa, Roma-Bari, Cariplo-Laterza, pp. 356-370.

Porta, P.L. (2007) 'Nota introduttiva', in P. Verri, Scritti di economia, finanza e amministrazione, a cura di Giuseppe Bognetti, Angelo Moioli, Pierluigi Porta, Giovanna Tonelli, tomo II (Edizione nazionale delle opere di Pietro Verri, vol. II), Roma, Edizioni di storia e letteratura, pp. 3-91.

Porta, P.L. e Scazzieri, R. (1999) 'Il contributo di Pietro Verri alla teoria economica. Società commerciale, società civile e governo dell'economia', in C. Capra (a cura di), Pietro Verri e il suo tempo, Milano, Cisalpino, tomo secondo, pp. 813-852.

Porta, P.L. e Scazzieri, R. (2002) 'Pietro Verri's Political Economy: Commercial Society, Civil Society, and the Science of the Legislator', History of Political Economy, 34 (1, Spring), pp. 83-110.

Quadrio Curzio, A. (2007) 'Cesare Beccaria e Pietro Verri: l'economia civile per il governo della "cosa pubblica" ', in A. Quadrio Curzio, Economisti ed economia. Per un'Italia europea: paradigmi tra il XVIII e il XX secolo, Bologna, Il Mulino, pp. 43-56.

Quadrio Curzio, A. e Pellizzari, F. (1999) Rent, Resources, Technology, Berlin, Springer.

Quadrio Curzio, A. e Scazzieri, R. (1985) Sui momenti costitutivi dell'economia politica, Bologna, Il Mulino.

Quadrio Curzio, A. e Scazzieri, R. (1986) 'Governo della moneta ed economia politica: su tre saggi di Cesare Beccaria e di Pietro Verri', in Sul disordine delle monete a Milano nel Settecento. Tre saggi di Cesare Beccaria e Pietro Verri, Milano, Electa, pp. 9- 43.

Quadrio Curzio, A. e Scazzieri, R. (1990) 'Profili di dinamica economica e strutturale', in A. Quadrio Curzio e R. Scazzieri, a cura di, Dinamica economica strutturale, Bologna, Il Mulino, pp. 11-51.

Quadrio Curzio, A. e Scazzieri, R. (2008) 'Historical Stylizations and Economic Theory', in R. Scazzieri, A. Sen e S. Zamagni (eds.), Markets, Money and Capital. Hicksian Perspectives for the Twenty-First Century, Cambridge, Cambridge University Press, pp. 185-203.

Scazzieri, R. (2008a) 'The Diffusion of Economic Ideas and the Formation of the Market Tradition', in R. Scazzieri e R. Simili, a cura di, The Migration of Ideas, Sagamore Beach, Science History Publications, pp. 73-97.

Scazzieri, R. (2008b) 'Bisogni umani, società civile e teoria del valore nelle lezioni di Luigi Valeriani (1807-1824)', in P. Barucci, L. Costabile e M. Di Matteo (a cura di), Gli archivi e la storia del pensiero economico, Bologna, Il Mulino, pp. 53-81.

Venturi, F. (1997) 'Nota introduttiva' a Cesare Beccaria, in F. Venturi, a cura di, Illuministi italiani, volume I, tomo I, Riformatori lombardi, piemontesi e toscani, pp. 3-26.

Venturi, F. (2001²) Utopia e riforma nell'illuminismo, Torino, Einaudi.

Verri, P. (1762) Il Gran Zoroastro, ossia Astrologiche osservazioni sui veri principi della scienza monetaria in soccorso della Risposta ad un amico, Lugano.

Verri, P. (1964 [1763]) Discorso sulla felicità, in P. Verri, Del piacere e del dolore ed altri scritti di filosofia ed economia, Milano, Feltrinelli, pp. 71-124.

Verri, P. 1986 a [1762]) Dialogo sul disordine delle monete nello Stato di Milano nel 
1762, in Sul disordine delle monete a Milano nel Settecento, cit., Milano, Electa per Banca del Monte di Milano.

Verri, P. (1986 b [1772]) Consulta sulle monete dello Stato di Milano di Pietro Verri presentata al Magistrato Camerale. Da inoltrarsi alla R.I. Corte, in Sul disordine delle monete a Milano nel Settecento, cit., Milano, Electa per Banca del Monte di Milano.

Verri, P. (2006 [1761-1763]) Considerazioni sul commercio dello Stato di Milano, in P. Verri, Scritti di economia, finanza e amministrazione, tomo I (Edizione nazionale delle opere di Pietro Verri, vol. II), Roma, Edizioni di storia e letteratura, pp. 107-345.

Verri, P. (2006 [1763]) 'Sul tributo del sale nello Stato di Milano', in P. Verri, Scritti di economia, finanza e amministrazione, tomo I (Edizione nazionale delle opere di Pietro Verri, vol. II), Roma, Edizioni di storia e letteratura, pp. 693-713.

Verri, P. (2007 [1769]) 'Sulle leggi vincolanti principalmente nel commercio dei grani. Riflessioni addattate allo Stato di Milano coll'occasione che trattasi di riformare il sistema d'annona', in P. Verri, Scritti di economia, finanza e amministrazione, tomo II (Edizione nazionale delle opere di Pietro Verri, vol. II), Roma, Edizioni di storia e letteratura, pp. 247-371.

Verri, P. (2007 [1771]) Meditazioni sulla economia politica, in P. Verri, Scritti di economia, finanza e amministrazione, tomo II (Edizione nazionale delle opere di Pietro Verri, vol. II), Roma, Edizioni di storia e letteratura, pp. 393-560. 